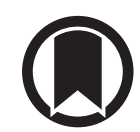

CrossMark

\title{
Circulating free DNA concentration is an independent prognostic biomarker in lung cancer
}

\begin{abstract}
Claire Tissot ${ }^{1,2}$, Anne-Claire Toffartt, ${ }^{3,4}$, Stéphanie Villar ${ }^{2}$, Pierre-Jean Souquet ${ }^{1}$, Patrick Merle ${ }^{5}$, Denis Moro-Sibilot ${ }^{3,4}$, Maurice Pérol $^{6}$, Jiri Zavadil $^{2}$, Christian Brambilla ${ }^{3,4}$, Magali Olivier ${ }^{2}$ and Sébastien Couraud ${ }^{1,7}$

Affiliations: ${ }^{1}$ Department of Acute Respiratory Medicine and Thoracic Oncology Department, Lyon Sud Hospital and Lyon University Cancer Institute, Lyon University Hospital, Pierre Bénite, France. ${ }^{2}$ International Agency for Research on Cancer, Molecular Mechanisms and Biomarkers Group, Lyon, France. ${ }^{3}$ Université Grenoble 1, INSERM, U 823, Institut A Bonniot, Université J Fourier, La Tronche, France. ${ }^{4}$ Thoracic Oncology Unit, Teaching Hospital A Michallon, Grenoble, France. ${ }^{5}$ Thoracic Oncology Unit, Clermont-Ferrand University Hospital, Clermont-Ferrand, France. ${ }^{6}$ Thoracic Oncology Unit, Lyon Cancer Centre Léon Bérard, Lyon, France. "EMR 3738 “Therapeutic Targeting in Oncology”, Lyon Sud - Charles Mérieux Faculty of Medicine, Lyon 1 University, Oullins, France.
\end{abstract}

Correspondence: Sébastien Couraud, Service de Pneumologie Aigue Spécialisée et Cancérologie Thoracique, CH Lyon Sud, Hospices Civils de Lyon, 165 Chemin du Grand Revoyet, 69495 Pierre Bénite Cedex, France.

E-mail: sebastien.couraudachu-lyon.fr

@ERSpublications

Total cell-free DNA is not associated with chemotherapy response in advanced nonsmall cell lung cancer http://ow.ly/Qpqyx

ABSTRACT Plasma circulating cell-free (cf)DNA is of interest in oncology because it has been shown to contain tumour DNA and may thus be used as liquid biopsy. In nonsmall cell lung cancer (NSCLC), cfDNA quantification has been proposed for the monitoring and follow-up of patients. However, available studies are limited and need to be confirmed by studies with larger sample sizes and including patients who receive more homogenous treatments. Our objective was to assess the predictive and prognostic value of plasma cfDNA concentration in a large series of patients with NSCLC and treated with a standard chemotherapy regimen.

We included samples from lung cancer patients recruited into the Pharmacogenoscan study. The cfDNA of 218 patients was extracted and quantified by fluorometry before and after two or three cycles of platinum-based chemotherapy. The association between baseline and post-chemotherapy concentrations and treatment response, assessed by RECIST (response evaluation criteria in solid tumours) or patient survival was analysed.

Patients with high cfDNA concentrations (highest tertile) at baseline had a significantly worse disease-free and overall survival than those with lower concentrations (lowest and middle tertiles) (median overall survival 10 months (95\% CI 10.7-13.9) versus 14.2 months (95\% CI 12.6-15.8), respectively; $\mathrm{p}=0.001$ ). In multivariate analysis, increased baseline concentration of cfDNA was an independent prognostic factor. However, we did not find any association between cfDNA concentration and response to treatment.

cfDNA may be a biomarker for the assessment of prognosis in NSCLC. However, total concentration of cfDNA does not appear to predict chemotherapy response.

For editorial comments see Eur Respir J 2015; 46: 1548-1550 [DOI: 10.1183/09031936.01482-2015]

This article has supplementary material available from erj.ersjournals.com

Received: April 302015 | Accepted after revision: July 052015 | First published online: Oct 222015

This study is registered at clinicaltrials.gov with identifier number NCT00222404.

Support statement: The Pharmacogenoscan study was supported by the French Ministry of Health (national hospital clinical research programme (PHRC) 2008). Claire Tissot was supported by a grant from Hospices Civils de Lyon (Année Recherche).

Conflict of interest: Disclosures can be found alongside the online version of this article at erj.ersjournals.com

Copyright OERS 2015 


\section{Introduction}

Lung cancer is the world leading cause of death by cancer [1]. It is usually diagnosed at an advanced or metastatic stage because local and regional dissemination remains asymptomatic for a long time in most patients. Prognosis of advanced lung cancer is very poor, with a 5-year survival of metastatic stages that does not exceed 5\% [2]. The main prognostic factors are disease stage, performance status, age at diagnosis, response to first-line chemotherapy, plasma lactate dehydrogenase level and presence of a somatic mutation or rearrangement in a gene or pathway for which targeted therapy is available (such as epidermal growth factor receptor (EGFR) or anaplastic lymphoma kinase) [3,4]. Treatments for advanced stages are based on cytotoxic chemotherapy and targeted therapies [5]. Response to treatment is assessed using computed tomography (CT) using the RECIST (response evaluation criteria in solid tumours) criteria [6]. To date, there is no reliable biomarker for the evaluation of response to standard chemotherapy treatment in lung cancer and there is a need for more prognostic factors to manage patients with advanced nonsmall cell lung cancer (NSCLC).

Circulating cell-free (cf)DNA has recently received renewed interest for its potential use as biomarker in oncology [7]. cfDNA corresponds to cell-free DNA fragments circulating in the bloodstream and that can be extracted from plasma or serum. It is believed to be released in the bloodstream through necrosis, apoptosis or active secretion by nucleated cells such as lymphocytes. cfDNA is mostly composed of constitutive genomic DNA. Several conditions may be associated with increasing concentrations of cfDNA, including inflammation, tissue trauma and cancer $[8,9]$. In the case of cancer, a significant proportion of cfDNA comes from tumour cells and cfDNA concentration reflects the tumour burden [10]. Moreover, cfDNA may originate from circulating tumour cells and thus may reflect the micro-metastatic disease and the aggressiveness potential of the disease [9].

It has been hypothesised that cfDNA may be a predictive factor of tumour response and a good candidate for a prognostic factor. In patients with lung cancer, some studies have shown that cfDNA concentration could be a predictive factor of tumour response to surgery and to radiation therapy $[11,12]$, but very few studies have investigated cfDNA concentration as a predictive factor of tumour response to chemotherapy. These studies included small number of samples and results are conflicting [13-15]. Studies of the prognostic value of cfDNA also show conflicting results [11, 13].

Here we assessed the predictive and prognostic values on tumour response and survival of cfDNA concentration in NSCLC patients treated with platinum-based chemotherapy.

\section{Materials and methods}

\section{Population}

Patients included in this study were from the French Pharmacogenoscan study. Pharmacogenoscan is a prospective cohort conducted in six hospitals in the Rhône-Alpes-Auvergne region of France. Its aim was to identify biological and histological factors associated with outcomes in patients with NSCLC. The primary objective was to identify associations between specific biomarkers and disease control rate. The secondary objective was to identify biomarkers associated with patient survival. All patients signed an informed consent form. First results of the Pharmacogenoscan study have been reported elsewhere [16, 17].

Inclusion criteria of the Pharmacogenoscan study were as follows. 1) Consecutive patients diagnosed with a NSCLC in one of the six participating centres between July 2005 and August 2010;2) cancer may be of any stage but with a willingness to be treated by platinum-based doublet chemotherapy as either neo-adjuvant treatment or first-line treatment for metastatic or recurrent disease (doublet drug was at investigator's choice); and 3) Eastern Cooperative Oncology Group (ECOG) performance status of 0-2 at inclusion [18].

All patients underwent blood sampling before any cancer treatment and at first response evaluation, which took place after two or three cycles of platinum-based chemotherapy at the investigator's choice. Response to chemotherapy was assessed using RECIST criteria [6].

From the Pharmacogenoscan dataset, we restricted inclusion to NSCLC patients with available plasma samples collected both before and after chemotherapy and with available RECIST response assessments [19]. In addition, we chose to restrict inclusion to stage IIIB-IV only. Indeed, we have shown previously that cfDNA concentration is associated with staging [10].

The Pharmacogenoscan study was approved by the ethics committee of the Grenoble University Hospital (Grenoble, France) (NCT00222404). The present ancillary study was approved by the International Agency for Research on Cancer (IARC) ethics committee (IEC 14-04).

\section{Plasma cfDNA}

Venous blood was collected in sterile EDTA-coated vials at baseline (before the first chemotherapy cycle) and at first CT scan response assessment (occurring after the second or third cycle of chemotherapy, 
depending on the centre, but 3 weeks after the last cycle in any case). Samples were centrifuged $(\sim 590 \times g$ for $15 \mathrm{~min}$ at room temperature) within $4 \mathrm{~h}$ of blood collection. Plasma was isolated and stored at $-80^{\circ} \mathrm{C}$. All plasma samples were stored centrally at Grenoble University Hospital. Samples used for the present study were shipped from Grenoble to IARC in dry ice according to international standards.

cfDNA was extracted from plasma samples using the QIAamp Circulating Nucleic Acid Kit (Qiagen, Valencia, CA, USA) following manufacturer instructions. Elution was performed in $50 \mu \mathrm{L}$ of ultra-pure water (molecular biology grade sterile water; Eppendorf, Hamburg, Germany), and the isolated cfDNA was kept at $-20^{\circ} \mathrm{C}$ before quantification.

Quantification of cfDNA was performed by fluorometry using PicoGreen dsDNA Kit (Molecular Probes, Eugene, OR, USA) following manufacturer instructions. Plates were read in a Fluoroscan Ascent FL Fluorometer (Thermo Fisher Scientific Oy, Vantaa, Finland) at wavelengths of $538 \mathrm{~nm}$ and $485 \mathrm{~nm}$ for emisison and excitation, respectively. Standard curves were obtained by serial dilution of the $\lambda$-DNA stock (provided by the manufacturer). Blank values were subtracted and plasma DNA concentrations were determined from the standard curve.

Researchers performing the DNA quantification were blinded to the patient's clinical outcomes.

\section{Statistical methods}

Hypothesis of a normal distribution was tested using the Kolmogorov-Smirnov test for all continuous variables. Normally distributed variables are presented as mean \pm SD; non-normally distributed variables are presented as median (interquartile range (IQR)). For comparisons of non-normal continuous variables we used the Wilcoxon test for two related samples and the Mann-Whitney U-test and the Kruskal-Wallis tests for two or more independent samples, respectively. Categorical variables are expressed as percentages. Correlations were assessed using Spearman's $\rho$.

For survival analysis we used the Kaplan-Meier method and median survival (and corresponding 95\% confidence intervals) were compared using the log-rank test. Then, we used a Cox regression model (enter method) in order to assess variables associated with survival. The model was first set in univariate analysis, then we adjusted the model with relevant variables and/or variables found to be significant in univariate analysis.

All tests were two-sided and $\mathrm{p}<0.05$ was considered significant. For the Cox model, $\mathrm{p}<0.1$ was used. Statistical analysis was performed using SPSS (version 19.0; IBM, Armonk, NY, USA).

\section{Results}

Population

Out of 537 patients recruited in the Pharmacogenoscan study between 2005 and 2010, 218 patients met the inclusion criteria for the present study (online supplementary fig. S1). This series of patients included 147 cases of adenocarcinoma, 43 cases of squamous cell carcinoma and 28 large cell carcinoma cases (table 1). The median follow-up time was 12.3 (15) months. Overall, 176 patients had stage IV disease and 42 patients had stage IIIB disease. At response evaluation no patients showed a complete response, 51 a partial response, 132 a stable disease and 35 a progressive disease, according to RECIST criteria.

\section{cfDNA concentrations and clinical correlates}

cfDNA concentration was measured by fluorometry in plasma samples collected before (baseline) and after chemotherapy treatment in 218 patients with NSCLC. cfDNA concentrations ranged from $2.54 \mathrm{ng} \cdot \mathrm{mL}^{-1}$ to $6451.36 \mathrm{ng} \cdot \mathrm{mL}^{-1}$. The median baseline cfDNA concentration (before chemotherapy) was 29.5 (37.0) ng. $\mathrm{mL}^{-1}$ and the median post-chemotherapy cfDNA concentration was $28.9(38.1) \mathrm{ng} \cdot \mathrm{mL}^{-1}$ $(\mathrm{p}=0.421)$. Baseline and post-chemotherapy concentrations were both significantly correlated (Spearman's $\left.\rho=0.459, \mathrm{p}<10^{-4}\right)$.

There was no correlation between sample conservation time (ranging from 2006 to 2010) and baseline or final cfDNA concentrations (Spearman's $\rho=-0.022, p=0.746$; and $\rho=-0.045, p=0.507$, for baseline and final concentrations, respectively). Moreover, we found no significant difference in median concentrations according to year of sampling (not shown).

There was no statistically significant association between cfDNA concentrations (both baseline and post-chemotherapy) and patients or tumour characteristics, including body mass index at diagnosis, TNM (tumour, node and metastases) stage (IIIB versus IV) or histological type (data not shown). However, we found a positive association between baseline cfDNA concentration and ECOG performance status at diagnosis (median (IQR) 24.19 (21.86) ng.mL ${ }^{-1}, 34.76(61.74) \mathrm{ng} \cdot \mathrm{mL}^{-1}$ and 108.44 (3307.37) ng.mL $\mathrm{mL}^{-1}$ for performance status 0,1 and 2 , respectively; $\mathrm{p}=0.001$ ). Interestingly, both variables were correlated, albeit modestly $\left(\rho=0.239, p<10^{-4}\right)$. 


\section{TABLE 1 Main characteristics of patients included in the study}

\begin{tabular}{|c|c|}
\hline Subjects & 218 \\
\hline \multicolumn{2}{|l|}{ Sex } \\
\hline Male & $163(74.8)$ \\
\hline Female & $55(25.2)$ \\
\hline Age at diagnosis years & $59.9 \pm 9.0$ \\
\hline \multicolumn{2}{|c|}{ Weight loss percentage of usual weight } \\
\hline$\leqslant 5 \%$ & $121(55.5)$ \\
\hline$>5 \%$ and $\leqslant 10 \%$ & $48(22.0)$ \\
\hline$>10 \%$ & $38(17.4)$ \\
\hline \multicolumn{2}{|l|}{ BMI categories at diagnosis } \\
\hline Underweight $\left(<18 \mathrm{~kg} \cdot \mathrm{m}^{-2}\right)$ & 18 (8.3) \\
\hline Normal weight $\left(18-25 \mathrm{~kg} \cdot \mathrm{m}^{-2}\right)$ & $126(57.8)$ \\
\hline Pre-obesity $\left(25-30 \mathrm{~kg} \cdot \mathrm{m}^{-2}\right)$ & 56 (25.7) \\
\hline Obesity $\left(>30 \mathrm{~kg} \cdot \mathrm{m}^{-2}\right)$ & $17(7.8)$ \\
\hline \multicolumn{2}{|l|}{ Histological subtype } \\
\hline Adenocarcinoma & $147(67.4)$ \\
\hline Squamous carcinoma & 43 (19.7) \\
\hline Large cell carcinoma & $28(12.8)$ \\
\hline \multicolumn{2}{|l|}{ Performance status at diagnosis } \\
\hline 0 & 85 (38.9) \\
\hline 1 & $128(58.7)$ \\
\hline 2 & 5 (2.3) \\
\hline \multicolumn{2}{|l|}{ Stage at diagnosis } \\
\hline Stage IV & $176(80.7)$ \\
\hline Stage IIIB & 42 (19.3) \\
\hline \multicolumn{2}{|l|}{ Platin drug } \\
\hline Cisplatin & 185 (84.9) \\
\hline Carboplatin & $33(15.1)$ \\
\hline \multicolumn{2}{|l|}{ Doublet drug } \\
\hline Vinorelbine & 25 (11.5) \\
\hline Pemetrexed & $39(17.9)$ \\
\hline Paclitaxel & $28(12.8)$ \\
\hline Gemcitabine & 78 (35.8) \\
\hline Docetaxel & $48(22.0)$ \\
\hline Bevacizumab use with CT & $11(5.0)$ \\
\hline \multicolumn{2}{|c|}{ Response category after platin-based chemotherapy (RECIST) } \\
\hline Progressive disease & $35(16.1)$ \\
\hline Stable disease & $132(60.6)$ \\
\hline Partial response & $51(23.4)$ \\
\hline \multicolumn{2}{|c|}{ Number of chemotherapy line(s) after the first line $(n=216)$} \\
\hline None & 45 (20.8) \\
\hline 1 & 68 (31.5) \\
\hline 2 & $57(26.4)$ \\
\hline$\geqslant 3$ & $46(21.3)$ \\
\hline
\end{tabular}

Data are presented as $\mathrm{n}, \mathrm{n}(\%)$ or mean \pm SD. BMI: body mass index; CT: computed tomography; RECIST: response evaluation criteria in solid tumours.

cfDNA concentrations and tumour response in advanced NSCLC

Considering three groups of tumour response (partial response versus stable disease versus progressive disease), no significant difference in the median concentrations of cfDNA (both baseline and post-chemotherapy concentrations) was observed between groups (table 2). Moreover, there was no significant difference in the variation of cfDNA during treatment (difference between post-chemotherapy and baseline concentration) between the three groups of tumour response (table 2). When considering objective response, thus comparing stable disease plus partial response versus progressive disease, no association was found. In addition, we divided the population into three tertiles according to their baseline cfDNA concentration (cut-off values $\leqslant 22.26 \mathrm{ng} \cdot \mathrm{mL}^{-1}, 22.27-42.12 \mathrm{ng} \cdot \mathrm{mL}^{-1}$ and $>42.12 \mathrm{ng} \cdot \mathrm{mL}^{-1}$ ). Again, there was no significant difference between baseline and post-chemotherapy cfDNA concentrations according to tumour response across tertiles (fig. 1). Finally, we also used the RECIST response as a continuous variable (percentage) and found no significant association or correlation with both baseline and post-chemotherapy cfDNA concentrations (Spearman's $\rho=0.17, p=0.806$ and $-0.12, p=0.861$ for correlation between RECIST (percentage) and baseline or post-chemotherapy, respectively). 


\begin{tabular}{|c|c|c|c|c|}
\hline & $\begin{array}{c}\text { Progressive } \\
\text { disease }\end{array}$ & $\begin{array}{c}\text { Stable } \\
\text { disease }\end{array}$ & $\begin{array}{l}\text { Partial } \\
\text { response }\end{array}$ & p-value \\
\hline Baseline concentration $\mathrm{ng} \cdot \mathrm{mL}^{-1}$ & $23.88(35.84)$ & $32.83(37.32)$ & $26.79(28.98)$ & 0.358 \\
\hline $\begin{array}{l}\text { Post-chemotherapy } \\
\text { concentration } \mathrm{ng} \cdot \mathrm{mL}^{-1}\end{array}$ & $24.16(21.66)$ & $28.61(37.92)$ & $30.72(61.33)$ & 0.358 \\
\hline $\begin{array}{l}\text { Difference between } \\
\text { post-chemotherapy and baseline } \\
\text { concentration } \mathrm{ng} \cdot \mathrm{mL}^{-1}\end{array}$ & $-0.22(27.52)$ & $-2.01(28.63)$ & $-0.56(41.95)$ & 0.473 \\
\hline Variation in concentration $\%$ & $-0.01(1.04)$ & $-0.08(0.92)$ & $-0.02(1.80)$ & 0.402 \\
\hline
\end{tabular}

Data are presented as median (interquartile range), unless otherwise stated.

\section{Prognostic value of cfDNA concentration}

The median progression-free survival (PFS) was 6.9 months (95\% CI 6.1-7.7 months) and overall survival was 12.3 months (95\% CI 10.7-13.9 months). The highest tertile group of the baseline cfDNA concentrations showed a significantly shorter PFS than the two other tertiles taken together (5.7 months, 95\% CI 4.5-6.9 months versus 7 months, 95\% CI 6.3-7 months, respectively; $\mathrm{p}=0.034$ ). This prognostic value of baseline cfDNA concentration was also significant when taking overall survival as outcome variable: 10 months (95\% CI 10.7-13.9 months) in the highest tertile versus 14.2 months (95\% CI 12.6 15.8 months) in the low/middle tertile groups ( $\mathrm{p}=0.001$ ) (fig. 2).

In univariate analysis we found disease stage, performance status at diagnosis and response rate to be associated with both overall survival and PFS (online supplementary table S1).These variables and patient age were used in a multivariate Cox regression model (table 3). The prognostic value of baseline cfDNA concentration remained significant in this multivariate analysis, with a significant association for overall survival and a trend for significance for PFS. For overall survival, the corresponding adjusted hazard ratio was 100030 (95\% CI $100003-100057, \mathrm{p}=0.028$ ), meaning that the risk of death increased by $0.003 \%$ for each increment of $1 \mathrm{ng} \cdot \mathrm{mL}^{-1}$ in baseline cfDNA concentration, independently of stage, performance status, age and response rate.

However, we found no significant association between survival outcomes and post-chemotherapy concentration of cfDNA (table 3).

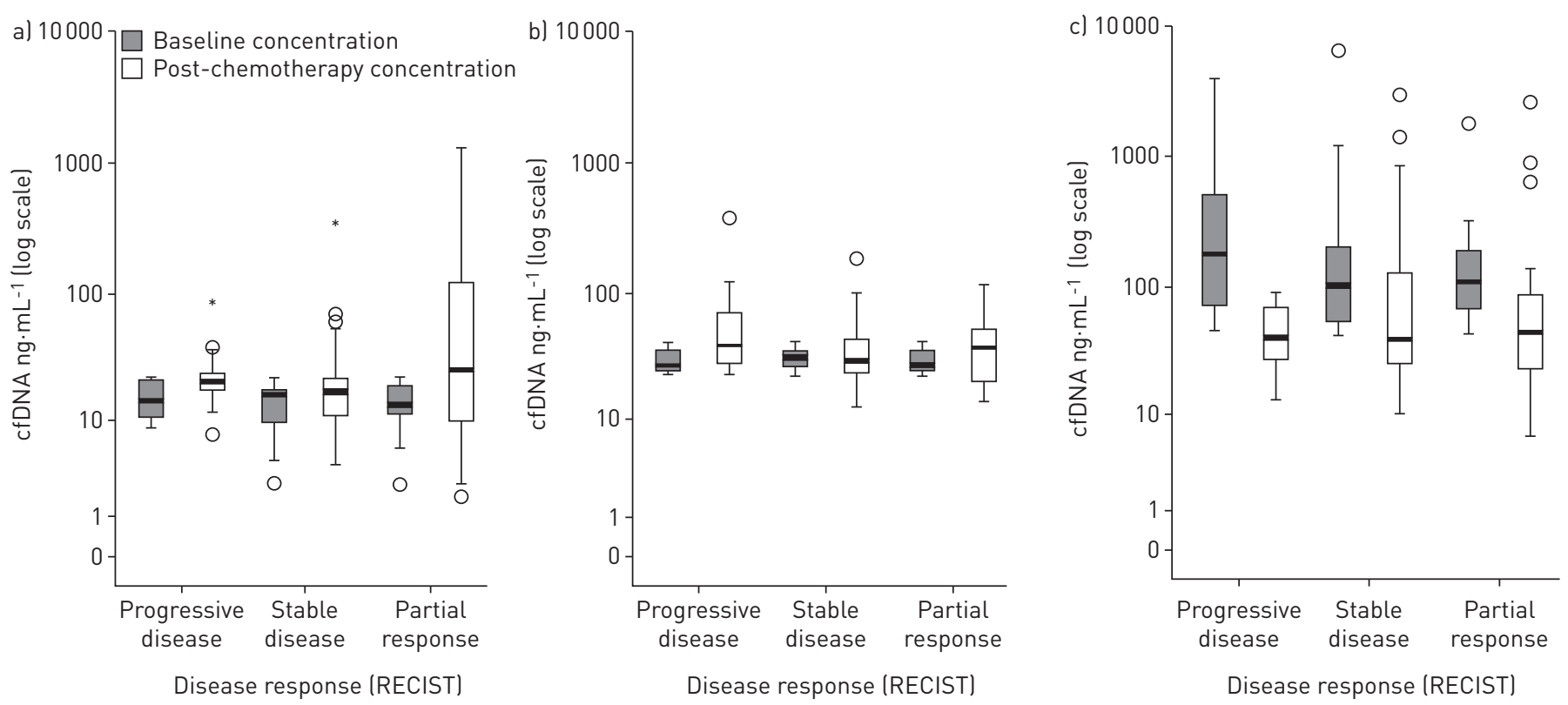

FIGURE 1 Box plots showing baseline and post-chemotherapy cell-free (cf)DNA concentrations according to tertile of baseline concentration and to response evaluation criteria in solid tumours (RECIST) response categories. a) First tertile; b) second tertile; c) third tertile. 

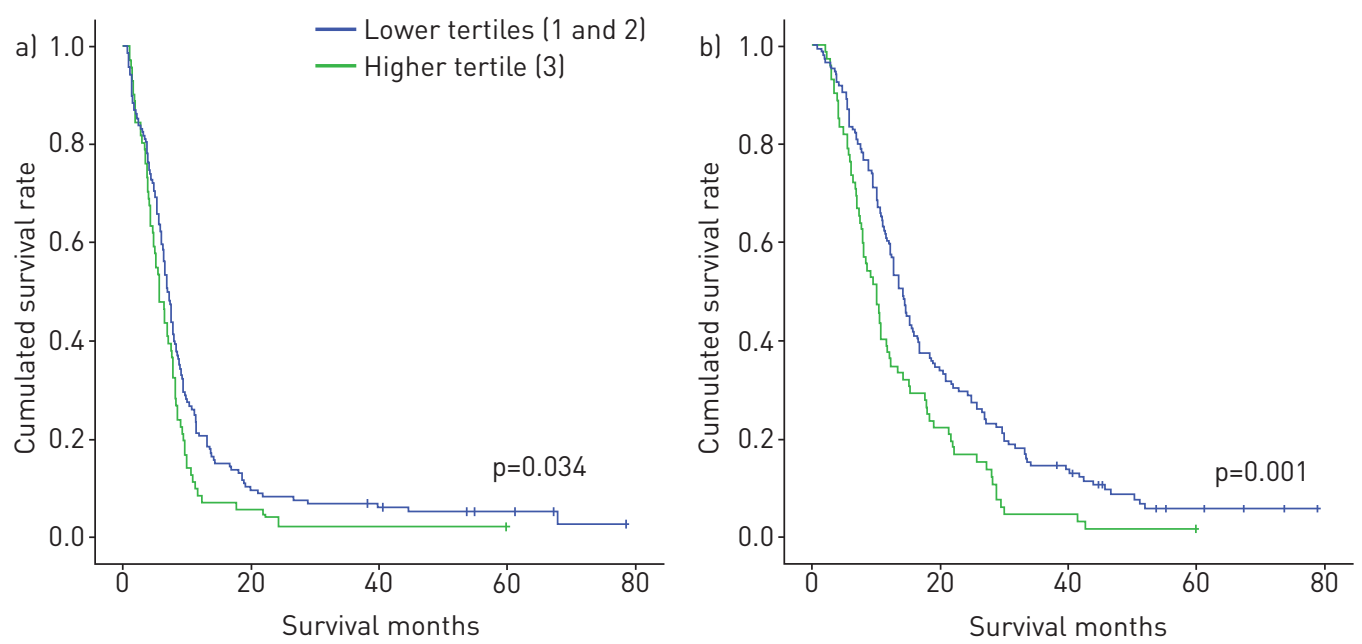

FIGURE 2 Survival curves showing a) progression-free and b) overall survival of patients classified by cell-free DNA concentration (highest versus two lowest tertiles).

\section{Discussion}

In this study, baseline cfDNA concentration in plasma has an independent prognostic value in NSCLC. This is in agreement with previous reports. Indeed, although some studies did not find any correlation between cfDNA levels and survival $[11,15,20]$, most reports showed a correlation between an elevated plasma cfDNA concentration and poor survival [13, 21-24]. GAUTSCHI et al. [13] found that an elevated baseline cfDNA concentration $\left(>10 \mathrm{ng} \cdot \mathrm{mL}^{-1}\right)$ was significantly associated with overall survival in multivariate analysis, independently of age, stage and history of treatment. In addition, three studies [14, $22,25]$ found very similar results to ours, using tertile subgroups. Our results thus provide further evidence, from a large series of patients, of the prognostic value of baseline cfDNA concentration.

In contrast, cfDNA concentration (baseline, post-chemotherapy or its variation during treatment) was not associated with response to chemotherapy. Previous studies have reported a significant association between cfDNA concentrations and response to treatment where cfDNA levels decreased after an efficient surgical treatment or treatment by radiotherapy [11, 12]. Here, we found that cfDNA concentrations did not correlate with RECIST response after two or three courses of chemotherapy treatment. Only three studies including patients treated with chemotherapy have been reported in the field of lung cancer (table 4). Our results are consistent with two of them [13, 14]. LeE et al. [14] also divided the population analysed into tertiles and found results similar to ours. Considering individual cfDNA variation during treatment, as GAUTSCHI et al. [13] did, we found that cfDNA was not reliable enough for response prediction: in some patients decreases in cfDNA levels can occur despite disease progression, whereas increases in cfDNA levels can occur despite response to the chemotherapy. Thus, this seems to confirm that cfDNA concentration does not predict treatment response in NSCLC when treatment is chemotherapy.

cfDNA is not specific to neoplastic conditions. It may also be elevated in inflammatory and infectious conditions. Thus, association between high concentration of cfDNA and poor prognosis may be linked to tumour burden and/or comorbidities [6]. However, our results underline the limitations of the analysis of the overall amount of cfDNA. Indeed, changes in cfDNA overall concentration may reflect not only changes

\begin{tabular}{|c|c|c|c|c|}
\hline & OR $(95 \% \mathrm{CI})$ & p-value & Adjusted OR $\mathrm{R}^{\#}(95 \% \mathrm{Cl})$ & p-value \\
\hline \multicolumn{5}{|l|}{ PFS } \\
\hline Baseline concentration $\llbracket$ & $1.0004(1.0002-1.0006)$ & 0.001 & 1.00025 (0.99998-1.00052) & 0.072 \\
\hline Post-chemotherapy concentration & $1.0001(0.9997-1.0005)$ & 0.636 & $1.00005(0.99966-1.00044)$ & 0.800 \\
\hline \multicolumn{5}{|l|}{ Overall survival } \\
\hline
\end{tabular}


TABLE 4 Cell-free (cf)DNA as a tool to monitor the response to treatment

\begin{tabular}{|c|c|c|c|c|c|c|c|}
\hline \multirow[t]{3}{*}{ First author [ref.] } & \multirow[t]{3}{*}{ Method } & \multirow[t]{3}{*}{ Sample } & \multirow[t]{3}{*}{ Subjects } & \multicolumn{4}{|c|}{ cfDNA concentration $\mathrm{ng} \cdot \mathrm{mL}^{-1}$} \\
\hline & & & & \multirow{2}{*}{$\begin{array}{l}\text { Before } \\
\text { treatment }\end{array}$} & \multicolumn{3}{|c|}{ After treatment } \\
\hline & & & & & Progressive disease & Stable disease & Partial response \\
\hline KUMAR [15] & PicoGreen & Plasma & 42 & $95 \pm 32.1$ & 159.7 & 101.1 & 51.7 \\
\hline \multirow[t]{2}{*}{ GAUTSCHI [13] } & Quantitative PCR & Serum & 91 & 39 & Increased & & \\
\hline & & Plasma & & 3.7 & & Decreased & Decreased \\
\hline LeE [14] & & & & Tertile 3 & $-18.5 \%$ & $-9.5 \%$ & $-18.6 \%$ \\
\hline
\end{tabular}

Data are presented as $\mathrm{n}$ or mean $\pm \mathrm{SD}$, unless otherwise stated.

in circulating tumour (ct)DNA, but other medical conditions which may lead to an increase in cfDNA, including chemotherapy that induces lysis in normal cells and thus promotes the release of normal cfDNA into the bloodstream. This may explain why overall concentration of cfDNA may be not predictive of response to chemotherapy. Focusing on ctDNA (which can be detected by the presence of genetic and epigenetic alterations that are specific to the primary tumour) might be a more adequate surrogate marker. For example, Newman et al. [20] recently reported a method called CAPP-Seq (cancer personalised profiling by deep sequencing) that allows the sensitive detection of multiple classes of somatic alterations and the quantification of ctDNA. Another study, by MARCQ et al. [26] showed that monitoring EGFR mutations in plasma of patients undergoing targeted EGFR-tyrosine kinase inhibitor (TKI) treatment accurately assessed tumour response and may detect progression (and mechanism of progression) under treatment. However, further studies of ctDNA are required to prove its usefulness in clinical practice. Thus, our results highlight the poor performance of cfDNA total concentration in assessing response to chemotherapy treatment, although cfDNA remains a potentially interesting biomarker if focusing on its tumour-specific fraction.

To our knowledge, our study is the largest in the field of lung cancer (tumour response and survival). Moreover, it includes a homogeneous population in terms of treatment, treatment response was assessed using RECIST criteria according to recommendations for NSCLC in clinical practice and the population analysed here was a classical population of advanced NSCLC patients. Patients with early progression $(n=6)$ or death $(n=25)$ were not studied in Pharmacogenoscan and thus were not included in our study [16]. The potential bias that may result from this exclusion is expected to be negligible, given the small number of cases. It also should be noted that, in the present study, cfDNA quantification was performed using PicoGreen, a simple method that may be easily implemented in clinical practice. Although most other studies used the quantitative (q) real-time PCR quantification, we chose the PicoGreen method because a good and positive correlation has been observed between PicoGreen and qPCR assays [27]. Although qPCR has high sensitivity and high accuracy, it requires a specific automated system and remains expensive, whereas the PicoGreen method is a rapid, effective, reliable and inexpensive method for cfDNA quantification. It also demonstrates high limits of detection, making the technique appropriate for plasma cfDNA analysis [27, 28].

The median cfDNA concentrations obtained by this method and previously published data on NSCLC were coherent, although average concentrations were lower than those reported in other series, including one from our team $[13,14]$. Such differences in absolute concentrations may come from blood sample processing, plasma isolation and storage or DNA quantification methods, as well as from the heterogeneity of the population studied. In this work, plasma isolation was performed within $4 \mathrm{~h}$ of blood drawing and according to the same standard procedure in all participating centres, to limit possible contamination by DNA from blood cells. Furthermore, cfDNA extraction and quantification were centrally performed in one laboratory with standard protocols to limit bias in quantification across centres. Regarding the potential impact of sample conservation time (long-term freezing may alter the quality and quantity of cfDNA), we have checked that there was no correlation between sample conservation time and cfDNA concentration.

Total cfDNA concentration appears to be an independent prognostic factor in lung cancer. Despite the fact that total cfDNA concentration is not informative to assess the effectiveness of chemotherapy and that other validated prognostic factors already exist (such as disease stage or performance status $[3,4]$ ), cfDNA remains interesting in this setting. Indeed, following-up the fraction of mutated cfDNA for biomarkers such as EGFR during therapy with a kinase inhibitor appears to be particularly promising for assessing TKI response and to detect early disease progression [26]. The use of cfDNA as liquid biopsy is also very promising for noninvasive somatic molecular profiling either at baseline or at follow-up for re-sampling $[26,29,30]$. 
In conclusion, our data suggest that the quantification of cfDNA from plasma may be a useful noninvasive technique for clinical practice. In advanced NSCLC, high baseline cfDNA concentration in plasma is associated with poor prognosis independently of age, performance status, stage and response. However, total cfDNA is not associated with chemotherapy response. The utility of cfDNA, and especially of tumour-specific cfDNA, should be assessed in larger and routine-based trials.

\section{References}

1 Ferlay J, Soerjomataram I, Dikshit R, et al. Cancer incidence and mortality worldwide: sources, methods and major patterns in GLOBOCAN 2012. Int J Cancer 2015; 136: E359-E386.

2 Alberg AJ, Brock MV, Ford JG, et al. Epidemiology of lung cancer: diagnosis and management of lung cancer, 3rd ed: American College of Chest Physicians evidence-based clinical practice guidelines. Chest 2013; 143: e1S-e29S.

3 Ost DE, Yeung SC, Tanoue LT, et al. Clinical and organizational factors in the initial evaluation of patients with lung cancer: diagnosis and management of lung cancer, 3rd ed: American College of Chest Physicians evidence-based clinical practice guidelines. Chest 2013; 143: e121S-e141S.

4 Nana-Sinkam SP, Powell CA. Molecular biology of lung cancer: diagnosis and management of lung cancer, 3rd ed: American College of Chest Physicians evidence-based clinical practice guidelines. Chest 2013; 143: e30S-e39S.

5 Peters S, Adjei AA, Gridelli C, et al. Metastatic non-small-cell lung cancer (NSCLC): ESMO Clinical Practice Guidelines for diagnosis, treatment and follow-up. Ann Oncol 2012; 23: Suppl. 7, 56-64.

6 Eisenhauer EA, Therasse P, Bogaerts J, et al. New response evaluation criteria in solid tumours: revised RECIST guideline (version 1.1). Eur J Cancer 2009; 45: 228-247.

7 Ulivi P, Silvestrini R. Role of quantitative and qualitative characteristics of free circulating DNA in the management of patients with non-small cell lung cancer. Cell Oncol 2013; 36: 439-448.

8 Schwarzenbach H, Hoon DS, Pantel K. Cell-free nucleic acids as biomarkers in cancer patients. Nat Rev Cancer 2011; 11: 426-437.

9 Jung K, Fleischhacker M, Rabien A. Cell-free DNA in the blood as a solid tumor biomarker: a critical appraisal of the literature. Clin Chim Acta 2010; 411: 1611-1624.

10 Couraud S, Vaca-Paniagua F, Villar S, et al. Noninvasive diagnosis of actionable mutations by deep sequencing of circulating free DNA in lung cancer from never-smokers: a proof-of-concept study from BioCAST/IFCT-1002. Clin Cancer Res 2014; 20: 4613-4624.

11 Sozzi G, Conte D, Mariani L, et al. Analysis of circulating tumor DNA in plasma at diagnosis and during follow-up of lung cancer patients. Cancer Res 2001; 61: 4675-4678.

12 Leon SA, Shapiro B, Sklaroff DM, et al. Free DNA in the serum of cancer patients and the effect of therapy. Cancer Res 1977; 37: 646-650.

13 Gautschi O, Bigosch C, Huegli B, et al. Circulating deoxyribonucleic acid as prognostic marker in non-small-cell lung cancer patients undergoing chemotherapy. J Clin Oncol 2004; 22: 4157-4164.

14 Lee YJ, Yoon KA, Han JY, et al. Circulating cell-free DNA in plasma of never smokers with advanced lung adenocarcinoma receiving gefitinib or standard chemotherapy as first-line therapy. Clin Cancer Res 2011; 17: 5179-5187.

15 Kumar S, Guleria R, Singh V, et al. Plasma DNA level in predicting therapeutic efficacy in advanced nonsmall cell lung cancer. Eur Respir J 2010; 36: 885-892.

16 Toffart AC, Timsit JF, Couraud S, et al. Immunohistochemistry evaluation of biomarker expression in non-small cell lung cancer (Pharmacogenoscan study). Lung Cancer 2014; 83: 182-188.

17 Toffart AC, Moro-Sibilot D, Couraud S, et al. Evaluation of RECIST in chemotherapy-treated lung cancer: the Pharmacogenoscan Study. BMC Cancer 2014; 14: 989.

18 Oken MM, Creech RH, Tormey DC, et al. Toxicity and response criteria of the Eastern Cooperative Oncology Group. Am J Clin Oncol 1982; 5: 649-655.

19 American Joint Committee on Cancer. AJCC Cancer Staging Manual. 7th Edn. New York, Springer, 2009.

20 Newman AM, Bratman SV, To J, et al. An ultrasensitive method for quantitating circulating tumor DNA with broad patient coverage. Nat Med 2014; 20: 548-554.

21 Beau-Faller M, Gaub MP, Schneider A, et al. Plasma DNA microsatellite panel as sensitive and tumor-specific marker in lung cancer patients. Int J Cancer 2003; 105: 361-370.

22 van der Drift MA, Hol BE, Klaassen $\mathrm{CH}$, et al. Circulating DNA is a non-invasive prognostic factor for survival in non-small cell lung cancer. Lung Cancer 2010; 68: 283-287.

23 Fournié GJ, Courtin JP, Laval F, et al. Plasma DNA as a marker of cancerous cell death: investigations in patients suffering from lung cancer and in nude mice bearing human tumours. Cancer Lett 1995; 91: 221-227.

24 Sirera R, Bremnes RM, Cabrera A, et al. Circulating DNA is a useful prognostic factor in patients with advanced non-small cell lung cancer. $J$ Thorac Oncol 2011; 6: 286-290.

25 Sozzi G, Roz L, Conte D, et al. Plasma DNA quantification in lung cancer computed tomography screening: five-year results of a prospective study. Am J Respir Crit Care Med 2009; 179: 69-74.

26 Marcq M, Vallée A, Bizieux A, et al. Detection of EGFR mutations in the plasma of patients with lung adenocarcinoma for real-time monitoring of therapeutic response to tyrosine kinase inhibitors? J Thorac Oncol 2014; 9: e49-e50.

27 Szpechcinski A, Struniawska R, Zaleska J, et al. Evaluation of fluorescence-based methods for total $v s$. amplifiable DNA quantification in plasma of lung cancer patients. J Physiol Pharmacol 2008; 59: 675-681.

28 Gormally E, Caboux E, Vineis P, et al. Circulating free DNA in plasma or serum as biomarker of carcinogenesis: practical aspects and biological significance. Mutat Res 2007; 635: 105-117.

29 Qiu M, Wang J, Xu Y, et al. Circulating tumor DNA is effective for the detection of EGFR mutation in non-small cell lung cancer: a meta-analysis. Cancer Epidemiol Biomarkers Prev 2015; 24: 206-212.

30 Oxnard GR, Paweletz CP, Kuang Y, et al. Noninvasive detection of response and resistance in EGFR-mutant lung cancer using quantitative next-generation genotyping of cell-free plasma DNA. Clin Cancer Res 2014; 20 : $1698-1705$. 